\title{
ON CENTRAL AUTOMORPHISMS FIXING THE CENTER ELEMENT-WISE
}

\author{
MANOJ K. YADAV
}

\begin{abstract}
Let $G$ be a finite $p$-group of nilpotency class 2 . We find necessary and sufficient conditions on $G$ such that each central automorphism of $G$ fixes the center of $G$ element-wise.
\end{abstract}

\section{INTRODUCTION}

Let $G$ be a finite group. By Z $(G), \gamma_{2}(G)$, Aut $(G)$ and $\operatorname{Inn}(G)$, we denote the center, the commutator subgroup, the group of all automorphisms and the group of all inner automorphisms of $G$ respectively. For $x \in G,[G, x]$ denotes the set $\left\{[g, x]=g^{-1} x^{-1} g x \mid g \in G\right\}$. To say that some $\mathrm{H}$ is a subgroup (proper subgroup) of $G$ we write $\mathrm{H} \leq G(\mathrm{H}<G)$. For any group $\mathrm{H}$ and an abelian group K, $\mathrm{Hom}(\mathrm{H}, \mathrm{K})$ denotes the group of all homomorphisms from $\mathrm{H}$ to $\mathrm{K}$. An automorphism $\alpha$ of $G$ is called central if $x^{-1} \alpha(x) \in \mathrm{Z}(G)$ for all $x \in G$. The set of all central automorphisms of $G$, which is here denoted by $\operatorname{Autcent}(G)$, is a normal subgroup of $\operatorname{Aut}(G)$. Notice that Autcent $(G)=\mathrm{C}_{\mathrm{Aut}(G)}(\operatorname{Inn}(G))$, the centralizer of the subgroup $\operatorname{Inn}(G)$ in the group $\operatorname{Aut}(G)$.

Let $\mathrm{M}$ and $\mathrm{N}$ be two normal subgroups of $G$. By $\operatorname{Aut}^{\mathrm{N}}(G)$ we mean the subgroup of $\operatorname{Aut}(G)$ consisting of all the automorphisms which centralize $G / \mathrm{N}$ and by $\operatorname{Aut}_{\mathrm{M}}(G)$ we mean the subgroup of $\operatorname{Aut}(G)$ consisting of all the automorphisms which centralize M. We denote $\operatorname{Aut}^{\mathrm{N}}(G) \cap \operatorname{Aut}_{\mathrm{M}}(G)$ by $\operatorname{Aut}_{\mathrm{M}}^{\mathrm{N}}(G)$. Throughout the paper $p$ always denotes a prime number.

In a very recent article of Attar [2, it is proved for a finite $p$-group $G$ that $\operatorname{Aut}_{\mathrm{Z}(G)}^{\mathrm{Z}(G)}(G)=\operatorname{Inn}(G)$ if and only if $G$ is abelian or $G$ is nilpotent of class 2 and $\mathrm{Z}(G)$ is cyclic. So it may be interesting to study finite $p$-groups $G$ such that $\operatorname{Aut}_{\mathrm{Z}(G)}^{\mathrm{Z}(G)}(G)$ contains $\operatorname{Inn}(G)$ and coincides with $\operatorname{Autcent}(G)$. Notice that if $\operatorname{Inn}(G) \leq \operatorname{Autcent}(G)$, then the nilpotency class of $G$ is at most 2. Moreover, if $G$ is abelian with $\operatorname{Autcent}(G)=\operatorname{Aut}_{\mathrm{Z}(G)}^{\mathrm{Z}(G)}(G)$, then it follows that $G=1$. So we restrict our attention to finite $p$-groups of class 2 .

Let $G$ be a finite $p$-group of class 2. Then $G / \mathrm{Z}(G)$ and $\gamma_{2}(G)$ have equal exponent $p^{c}$ (say). Let

$$
G / \mathrm{Z}(G)=\mathrm{C}_{p^{a_{1}}} \times \mathrm{C}_{p^{a_{2}}} \times \cdots \times \mathrm{C}_{p^{a_{r}}},
$$

where $\mathrm{C}_{p^{a_{i}}}$ is a cyclic group of order $p^{a_{i}}, 1 \leq i \leq r$, and $a_{1} \geq a_{2} \geq \cdots \geq a_{r}>0$. Let $k$ be the largest integer between 1 and $r$ such that $a_{1}=a_{2}=\cdots=a_{k}=c$.

Date: November 8, 2018.

2000 Mathematics Subject Classification. 20D45, 20 D15.

Research supported by DST (SERC Division), the Govt. of INDIA. 
Notice that $k \geq 2$. Set $\overline{\mathrm{M}}=\mathrm{M} / \mathrm{Z}(G)=\mathrm{C}_{p^{a_{1}}} \times \cdots \times \mathrm{C}_{p^{a_{k}}}$. Let

$$
G / \gamma_{2}(G)=\mathrm{C}_{p^{b_{1}}} \times \mathrm{C}_{p^{b_{2}}} \times \cdots \times \mathrm{C}_{p^{b_{s}}}
$$

where $b_{1} \geq b_{2} \geq \cdots \geq b_{s}>0$, be a cyclic decomposition of $G / \gamma_{2}(G)$ such that $\overline{\mathrm{M}}$ is isomorphic to a subgroup of $\overline{\mathrm{N}}=\mathrm{N} / \gamma_{2}(G):=\mathrm{C}_{p^{b_{1}}} \times \mathrm{C}_{p^{b_{2}}} \times \cdots \times \mathrm{C}_{p^{b_{k}}}$. Using the above terminology we state our result in

Theorem. Let $G$ be a finite p-group of nilpotency class 2. Then $\operatorname{Autcent}(G)=$ $\mathrm{Aut}_{\mathrm{Z}(G)}^{\mathrm{Z}(G)}(G)$ if and only if $r=s,(G / \mathrm{Z}(G)) / \overline{\mathrm{M}} \cong\left(G / \gamma_{2}(G)\right) / \overline{\mathrm{N}}$ and the exponents of $\mathrm{Z}(G)$ and $\gamma_{2}(G)$ are equal.

A generalization of the main theorem of Attar [2] is given in Proposition 2.3.

As a consequence of these results, we derive the main theorem of Curran and McCaughan [4] in Corollary 2.6.

\section{Proofs}

Let $G$ be a finite group and $\mathrm{M}$ be a central subgroup of $G$. Let $\alpha \in \operatorname{Aut}^{\mathrm{M}}(G)$. Then we can define a homomorphism $f_{\alpha}$ from $G$ to M such that $f_{\alpha}(x)=x^{-1} \alpha(x)$. On the other hand, given a homomorphism $f$ from $G$ to M, we can always define an endomorphism $\alpha_{f}$ of $G$ such that $\alpha_{f}(x)=x f(x)$. But $\alpha_{f}$ is an automorphism of $G$ if and only if for every non-trivial element $m \in \mathrm{M}, f(m) \neq m^{-1}$.

The following lemma is an easy exercise.

Lemma 2.1. Let $G$ be a finite group and $\mathrm{M}$ be a central subgroup of $G$. Let $\mathrm{M} \leq \cap \operatorname{Ker}(f)$, where $f$ runs over all elements in $\operatorname{Hom}(G, \mathrm{M})$. Then the correspondence $f \rightarrow \alpha_{f}$ defined in the preceeding paragraph is a one-to-one mapping from $\operatorname{Hom}(G, \mathrm{M})$ onto $\mathrm{Aut}^{\mathrm{M}}(G)$. Moreover, this correspondence gives rise to a natural isomorphism between $\operatorname{Aut}_{\mathrm{Z}(G)}^{\mathrm{M}}(G)$ and $\operatorname{Hom}(G / \mathrm{Z}(G), \mathrm{M})$.

A finite group $G$ is said to be purely non-abelian if it does not have a non-trivial abelian direct factor. The following lemma is from [1].

Lemma 2.2. Let $G$ be a purely non-abelian finite group. Then the correspondence $\alpha \rightarrow f_{\alpha}$ defined above with $\mathrm{M}$ replaced by $\mathrm{Z}(G)$ is a one-to-one mapping of Autcent $(G)$ onto $\operatorname{Hom}\left(G / \gamma_{2}(G), \mathrm{Z}(G)\right)$.

The following proposition generalizes the main result of Attar [2].

Proposition 2.3. Let $G$ be a non-abelian finite p-group and $\mathrm{M}$ be a central subgroup of $G$. Then $\operatorname{Aut}_{\mathrm{Z}(G)}^{\mathrm{M}}(G)=\operatorname{Inn}(G)$ if and only if the nilpotency class of $G$ is $2, \gamma_{2}(G) \leq \mathrm{M}$ and $\mathrm{M}$ is cyclic.

Proof. Since $\mathrm{M} \leq \mathrm{Z}(G), f(m)=1$ for all $f \in \operatorname{Hom}(G / \mathrm{Z}(G), \mathrm{M})$ and $m \in \mathrm{M}$. Thus it follows from Lemma 2.1 that there is a natural isomorphism between $\operatorname{Aut}_{\mathrm{Z}(G)}^{\mathrm{M}}(G)$ and $\operatorname{Hom}(G / \mathrm{Z}(G), \mathrm{M})$. Now suppose that $\operatorname{Aut}_{\mathrm{Z}(G)}^{\mathrm{M}}(G)=\operatorname{Inn}(G)$. Since $\operatorname{Inn}(G)=$ $\mathrm{Aut}_{\mathrm{Z}(G)}^{\mathrm{M}}(G) \leq \operatorname{Autcent}(G)$, it follows that the nilpotency class of $G$ is at most 2 . As $G$ is non-abelian, it must be of class 2. Thus the exponents of $G / \mathrm{Z}(G)$ and $\gamma_{2}(G)$ are equal. Let $\left\{x_{1}, x_{2}, \cdots, x_{d}\right\}$ be a minimal generating set for $G$. Let $i_{j}$ denote the inner automorphism induced by $x_{j}$, i.e., $i_{j}(g)=x_{j}^{-1} g x_{j}$ for all $g \in G$, where $1 \leq$ $j \leq d$. So $g^{-1} i_{j}(g) \in \mathrm{M}$ for all $g \in G$ and $1 \leq j \leq d$, since $\operatorname{Inn}(G)=\operatorname{Aut}_{\mathrm{Z}(G)}^{\mathrm{M}}(G)$. But $g^{-1} i_{j}(g)=g^{-1} x_{j}^{-1} g x_{j}=\left[g, x_{j}\right]$. So $\left\{g^{-1} i_{j}(g) \mid g \in G\right\}=\left[G, x_{j}\right] \leq \mathrm{M}$. Since 
$\gamma_{2}(G)=\left\langle\left[G, x_{j}\right] \mid 1 \leq j \leq d\right\rangle$, it follows that $\gamma_{2}(G) \leq$ M. Therefore the exponent of $\mathrm{M}$ is greater than or equal to the exponent of $\gamma_{2}(G)$. Let the exponent of M be $p^{e}$. If possible, suppose that $\mathrm{M}$ is not cyclic. Then $\mathrm{M}=\mathrm{C}_{p^{e}} \times \mathrm{N}$, where $\mathrm{C}_{p^{e}}$ is a cyclic subgroup of order $p^{e}$ and $\mathrm{N}$ is some non-trivial proper subgroup of M. Now

$$
\begin{aligned}
|\operatorname{Hom}(G / \mathrm{Z}(G), \mathrm{M})| & =\left|\operatorname{Hom}\left(G / \mathrm{Z}(G), \mathrm{C}_{p^{e}}\right)\right||\operatorname{Hom}(G / \mathrm{Z}(G), \mathrm{N})|>|G / \mathrm{Z}(G)| \\
& =|\operatorname{Inn}(G)| .
\end{aligned}
$$

Thus $\left|\operatorname{Aut}_{\mathrm{Z}(G)}^{\mathrm{M}}(G)\right|=|\operatorname{Hom}(G / \mathrm{Z}(G), \mathrm{M})|>|\operatorname{Inn}(G)|$. This contradicts the given hypothesis. Hence M must be cyclic.

Conversely suppose that the nilpotency class of $G$ is $2, \gamma_{2}(G) \leq \mathrm{M}$ and $\mathrm{M}$ is cyclic. Then

$$
\left|\operatorname{Aut}_{\mathrm{Z}(G)}^{\mathrm{M}}(G)\right|=|\operatorname{Hom}(G / \mathrm{Z}(G), \mathrm{M})|=|G / \mathrm{Z}(G)|=|\operatorname{Inn}(G)|,
$$

since the exponent of $\mathrm{M}$ is at least equal to the exponent of $G / \mathrm{Z}(G)$. This completes the proof of the proposition.

The following lemma can be proved by a counting argument using Corollary 3.3 of [3]. But here we prove it by a direct argument.

Lemma 2.4. Let $G$ be a finite p-group such that $\operatorname{Autcent}(G)=\operatorname{Aut}_{\mathrm{Z}(G)}^{\mathrm{Z}(G)}(G)$. Then $G$ is purely non-abelian.

Proof. Assume contrarily that $G$ is not purely non-abelian. Then $G=\mathrm{H} \times \mathrm{A}$, where $\mathrm{H}$ is purely non-abelian and $\mathrm{A}$ is non-trivial abelian subgroup of $G$. Let $\left\{x_{1}, x_{2}, \cdots, x_{r}\right\}$ and $\left\{y_{1}, y_{2}, \cdots, y_{s}\right\}$ be minimal generating sets for $\mathrm{H}$ and $\mathrm{A}$ respectively. Then $S:=\left\{x_{1}, x_{2}, \cdots, x_{r}, y_{1}, y_{2}, \cdots, y_{s}\right\}$ is a minimal generating set for $G$. Obviously Z $(\mathrm{H}) \cap \Phi(G) \neq 1$, where $\Phi(G)$ denotes the Frattini subgroup of $G$. So we can always choose a non-trivial element $z \in \mathrm{Z}(\mathrm{H}) \cap \Phi(G)$ such that $z^{p}=1$. Define a map $f$ from $G$ to $G$ by $f(w)=w z$ for all $w \in S$. Now it follows from the lemma (there is only one lemma in the paper) of 5 that $f$ is an automorphism of $G$. Notice that $f \in \operatorname{Autcent}(G)$, but $f \notin \operatorname{Aut}_{\mathrm{Z}(G)}^{\mathrm{Z}(G)}(G)$. For, $f\left(y_{i}\right)=y_{i} z \neq y_{i}$, however $y_{i} \in \mathrm{Z}(G)$ for all $i$ such that $1 \leq i \leq s$. This contradicts the given hypothesis that Autcent $(G)=\operatorname{Aut}_{\mathrm{Z}(G)}^{\mathrm{Z}(G)}(G)$. Hence $G$ must be purely non-abelian. This completes the proof of the lemma.

Lemma 2.5. Let $\mathrm{A}$ and $\mathrm{B}$ be two finite abelian p-groups such that $\mathrm{A}=\mathrm{C}_{p^{a_{1}}} \times \mathrm{C}_{p^{a_{2}}}$ $\times \cdots \times \mathrm{C}_{p^{a_{s}}}$, where $a_{1} \geq a_{2} \geq \cdots \geq a_{s}>0$, and $\mathrm{B}=\mathrm{C}_{p^{b_{1}}} \times \mathrm{C}_{p^{b_{2}}} \times \cdots \times \mathrm{C}_{p^{b_{s}}}$, where $b_{1} \geq b_{2} \geq \cdots \geq b_{s}>0$. Let $b_{j} \geq a_{j}$ for all $j, 1 \leq j \leq s$, and $b_{j}>a_{j}$ for some such $j$. Let $t$ be the smallest integer between 1 and s such that $a_{j}=b_{j}$ for all $j$ such that $t+1 \leq j \leq s$. Then, for any finite abelian p-group $\mathrm{C},|\operatorname{Hom}(\mathrm{A}, \mathrm{C})|<|\operatorname{Hom}(\mathrm{B}, \mathrm{C})|$ if and only if the exponent of $\mathrm{C}$ is at least $p^{a_{t}+1}$.

Proof. Let $\mathrm{H}=\mathrm{C}_{p^{a_{1}}} \times \cdots \times \mathrm{C}_{p^{a_{t}}}, \mathrm{~K}=\mathrm{C}_{p^{b_{1}}} \times \cdots \times \mathrm{C}_{p^{b_{t}}}$ and $\mathrm{D} \cong \mathrm{C}_{p^{a} t+1} \times \cdots \times$ $\mathrm{C}_{p^{a_{s}}} \cong \mathrm{C}_{p^{b_{t+1}}} \times \cdots \times \mathrm{C}_{p^{b_{s}}}$, since $b_{j}=a_{j}$ for all $j$ such that $t+1 \leq j \leq s$. Then

$$
\operatorname{Hom}(\mathrm{A}, \mathrm{C}) \cong \operatorname{Hom}(\mathrm{H} \times \mathrm{D}, \mathrm{C}) \cong \operatorname{Hom}(\mathrm{H}, \mathrm{C}) \times \operatorname{Hom}(\mathrm{D}, \mathrm{C})
$$

and

$$
\operatorname{Hom}(\mathrm{B}, \mathrm{C}) \cong \operatorname{Hom}(\mathrm{K} \times \mathrm{D}, \mathrm{C}) \cong \operatorname{Hom}(\mathrm{K}, \mathrm{C}) \times \operatorname{Hom}(\mathrm{D}, \mathrm{C})
$$


Thus $|\operatorname{Hom}(\mathrm{A}, \mathrm{C})|<|\operatorname{Hom}(\mathrm{B}, \mathrm{C})|$ if and only if $|\operatorname{Hom}(\mathrm{H}, \mathrm{C})|<|\operatorname{Hom}(\mathrm{K}, \mathrm{C})|$. Since $b_{j} \geq a_{j}$, we have $|\operatorname{Hom}(\mathrm{H}, \mathrm{C})|<|\operatorname{Hom}(\mathrm{K}, \mathrm{C})|$ if and only if $\left|\operatorname{Hom}\left(\mathrm{H}, \mathrm{C}_{p^{c}}\right)\right|<$ $\left|\operatorname{Hom}\left(\mathrm{K}, \mathrm{C}_{p^{c}}\right)\right|$ for, at least, one cyclic group $\mathrm{C}_{p^{c}}$ which appears in the cyclic decomposition of $\mathrm{C}$. Notice that $\left|\operatorname{Hom}\left(\mathrm{H}, \mathrm{C}_{p^{c}}\right)\right|=\left|\operatorname{Hom}\left(\mathrm{K}, \mathrm{C}_{p^{c}}\right)\right|$ if $c \leq a_{t}$. Hence $\left|\operatorname{Hom}\left(\mathrm{H}, \mathrm{C}_{p^{c}}\right)\right|<\left|\operatorname{Hom}\left(\mathrm{K}, \mathrm{C}_{p^{c}}\right)\right|$ if and only if $c>a_{t}$. Since the exponent of $\mathrm{C}$ is at least $c$, this completes the proof of the lemma.

Now we are ready to prove our theorem which is stated in Section 1. In the proof, we use the same terminology as we used in the statement of the theorem.

Proof of the theorem. We have $G / \mathrm{Z}(G)=\mathrm{C}_{p^{a_{1}}} \times \mathrm{C}_{p^{a_{2}}} \times \cdots \times \mathrm{C}_{p^{a_{r}}}$ and $G / \gamma_{2}(G)=\mathrm{C}_{p^{b_{1}}} \times \mathrm{C}_{p^{b_{2}}} \times \cdots \times \mathrm{C}_{p^{b_{s}}}$. Since $G / \mathrm{Z}(G)$ is a quotient group of $G / \gamma_{2}(G)$, we get $r \leq s$ and $b_{j} \geq a_{j}$ for all $j$ such that $1 \leq j \leq r$.

Suppose that Autcent $(G)=\operatorname{Aut}_{\mathrm{Z}(G)}^{\mathrm{Z}(G)}(G)$. Then $G$ is purely non-abelian by Lemma 2.4. Thus by Lemma 2.2 there is a one-to-one correspondence between Autcent $(G)$ and $\operatorname{Hom}\left(G / \gamma_{2}(G), \mathrm{Z}(G)\right.$ ). Also by Lemma 2.1 (with $\mathrm{M}=\mathrm{Z}(G)$ ) there is a natural isomorphism between $\operatorname{Aut}_{\mathrm{Z}(G)}^{\mathrm{Z}(G)}(G)$ and $\operatorname{Hom}(G / \mathrm{Z}(G), \mathrm{Z}(G))$.

First we claim that $r=s$. Suppose, if possible, that $r<s$. Since $b_{j} \geq a_{j}>0$ for all $j$ such that $1 \leq j \leq r$ and $b_{j}>0$ for $r+1 \leq j \leq s$, we have

$$
\begin{aligned}
&|\operatorname{Hom}(G / \mathrm{Z}(G), \mathrm{Z}(G))|=\left|\operatorname{Hom}\left(\mathrm{C}_{p^{a_{1}}} \times \cdots \times \mathrm{C}_{p^{a_{r}}}, \mathrm{Z}(G)\right)\right| \\
& \leq\left|\operatorname{Hom}\left(\mathrm{C}_{p^{b_{1}}} \times \cdots \times \mathrm{C}_{p^{b_{r}}}, \mathrm{Z}(G)\right)\right| \\
&<\left|\operatorname{Hom}\left(\mathrm{C}_{p^{b_{1}}} \times \cdots \times \mathrm{C}_{p^{b_{r}}}, \mathrm{Z}(G)\right)\right| \\
&\left|\operatorname{Hom}\left(\mathrm{C}_{p^{b_{r}+1}} \times \cdots \times \mathrm{C}_{p^{b_{s}}}, \mathrm{Z}(G)\right)\right| \\
&=\left|\operatorname{Hom}\left(\mathrm{C}_{p^{b_{1}}} \times \cdots \times \mathrm{C}_{p^{b_{s}}}, \mathrm{Z}(G)\right)\right| \\
&=\left|\operatorname{Hom}\left(G / \gamma_{2}(G), \mathrm{Z}(G)\right)\right| .
\end{aligned}
$$

This proves that $\operatorname{Aut}_{\mathrm{Z}(G)}^{\mathrm{Z}(G)}(G)<\operatorname{Autcent}(G)$. But this contradicts our supposition. Hence $r=s$ and our claim is true. This obviously gives the inequalities $b_{j} \geq a_{j}$ for all $j$ such that $1 \leq j \leq s$.

Now we claim that $(G / \mathrm{Z}(G)) / \overline{\mathrm{M}} \cong\left(G / \gamma_{2}(G)\right) / \overline{\mathrm{N}}$. Let us suppose contrary, i.e, $(G / \mathrm{Z}(G)) / \overline{\mathrm{M}} \not\left(G / \gamma_{2}(G)\right) / \overline{\mathrm{N}}$. Then $b_{j}>a_{j}$ for some $j$ such that $k+1 \leq j \leq s$. For, if $b_{j}=a_{j}$ for all such $j$, then $\mathrm{A}:=\mathrm{C}_{p^{a_{k+1}}} \times \cdots \times \mathrm{C}_{p^{a_{s}}}$ is isomorphic to $\mathrm{B}:=\mathrm{C}_{p^{b_{k+1}}} \times \cdots \times \mathrm{C}_{p^{b_{s}}}$ and therefore $(G / \mathrm{Z}(G)) / \overline{\mathrm{M}} \cong \mathrm{A} \cong \mathrm{B} \cong\left(G / \gamma_{2}(G)\right) / \overline{\mathrm{N}}$, which we are not taking. Let $t$ be the smallest integer between $k+1$ and $s$ such that $b_{j}=a_{j}$ for all $j$ satisfying $t+1 \leq j \leq s$. From our choice of $k$ we have $p^{c}>p^{a_{k+1}} \geq p^{a_{t}}$, where $p^{c}$ is the exponent of both $G / \mathrm{Z}(G)$ as well as $\gamma_{2}(G)$. Since the exponent of $\mathrm{Z}(G)$ is greater than or equal to $p^{c}$, it now follows that the exponent of $\mathrm{Z}(G)$ is at least $p^{a_{t}+1}$. Now applying Lemma 2.5 with $\mathrm{C}=\mathrm{Z}(G)$, we get $|\operatorname{Hom}(\mathrm{A}, \mathrm{Z}(G))|<|\operatorname{Hom}(\mathrm{B}, \mathrm{Z}(G))|$. So

$$
\begin{aligned}
|\operatorname{Hom}(G / \mathrm{Z}(G), \mathrm{Z}(G))| & =\mid \operatorname{Hom}(\overline{\mathrm{M}} \times \mathrm{A}, \mathrm{Z}(G)|=| \operatorname{Hom}(\overline{\mathrm{M}}, \mathrm{Z}(G))|| \operatorname{Hom}(\mathrm{A}, \mathrm{Z}(G)) \mid \\
& <|\operatorname{Hom}(\overline{\mathrm{N}}, \mathrm{Z}(G))||\operatorname{Hom}(\mathrm{B}, \mathrm{Z}(G))|=\mid \operatorname{Hom}(\overline{\mathrm{N}} \times \mathrm{B}, \mathrm{Z}(G) \mid \\
& =\left|\operatorname{Hom}\left(G / \gamma_{2}(G), \mathrm{Z}(G)\right)\right|,
\end{aligned}
$$


since $|\operatorname{Hom}(\overline{\mathrm{M}}, \mathrm{Z}(G))| \leq|\operatorname{Hom}(\overline{\mathrm{N}}, \mathrm{Z}(G))|$. Thus $\operatorname{Aut}_{\mathrm{Z}(G)}^{\mathrm{Z}(G)}(G)<\operatorname{Autcent}(G)$. This again contradicts our supposition. Hence $(G / \mathrm{Z}(G)) / \overline{\mathrm{M}} \cong\left(G / \gamma_{2}(G)\right) / \overline{\mathrm{N}}$.

Finally suppose that the exponent of $\mathrm{Z}(G)$ is not equal to the exponent of $\gamma_{2}(G)$. Thus the exponent of $\mathrm{Z}(G)$ is at least $p^{c+1}$, since the exponent of $\gamma_{2}(G)$ is $p^{c}$. Also $|\mathrm{Z}(G)|>\left|\gamma_{2}(G)\right|$ and therefore $|G / \mathrm{Z}(G)|<\left|G / \gamma_{2}(G)\right|$. Since $(G / \mathrm{Z}(G)) / \overline{\mathrm{M}} \cong$ $\left(G / \gamma_{2}(G)\right) / \overline{\mathrm{N}}$, it follows that $|\overline{\mathrm{N}}|>|\overline{\mathrm{M}}|$. Since $\overline{\mathrm{N}}=\mathrm{C}_{p^{b_{1}}} \times \cdots \times \mathrm{C}_{p^{b_{k}}}$ and $\overline{\mathrm{M}}=$ $\mathrm{C}_{p^{a_{1}}} \times \cdots \times \mathrm{C}_{p^{a_{k}}}$, where $b_{j} \geq a_{j}$ and $a_{j}=c$ for all $j$ such that $1 \leq j \leq k$, we conclude that $b_{j}>a_{j}$ for some such $j$. Let $t$ be the smallest integer between 1 and $k$ such that $b_{j}=a_{j}=c$ for all $j$ satisfying $t+1 \leq j \leq k$. We know that the exponent of $\mathrm{Z}(G)$ is at least $p^{c+1}=p^{a_{t}+1}$. So applying Lemma 2.5] with $\mathrm{A}=\overline{\mathrm{M}}, \mathrm{B}=\overline{\mathrm{N}}$ and $\mathrm{C}=\mathrm{Z}(G)$, we get $|\operatorname{Hom}(\overline{\mathrm{M}}, \mathrm{Z}(G))|<|\operatorname{Hom}(\overline{\mathrm{N}}, \mathrm{Z}(G))|$. Let $\mathrm{D} \cong \mathrm{C}_{p^{a_{k+1}}} \times \cdots \times \mathrm{C}_{p^{a_{s}}} \cong \mathrm{C}_{p^{b_{k+1}}} \times \cdots \times \mathrm{C}_{p^{b_{s}}}$. So

$$
\begin{aligned}
|\operatorname{Hom}(G / \mathrm{Z}(G), \mathrm{Z}(G))| & =|\operatorname{Hom}(\overline{\mathrm{M}} \times \mathrm{D}, \mathrm{Z}(G))|=|\operatorname{Hom}(\overline{\mathrm{M}}, \mathrm{Z}(G))||\operatorname{Hom}(\mathrm{D}, \mathrm{Z}(G))| \\
& <|\operatorname{Hom}(\overline{\mathrm{N}}, \mathrm{Z}(G))||\operatorname{Hom}(\mathrm{D}, \mathrm{Z}(G))|=|\operatorname{Hom}(\overline{\mathrm{N}} \times \mathrm{D}, \mathrm{Z}(G))| \\
& =\left|\operatorname{Hom}\left(G / \gamma_{2}(G), \mathrm{Z}(G)\right)\right| .
\end{aligned}
$$

Thus $\operatorname{Aut}_{\mathrm{Z}(G)}^{\mathrm{Z}(G)}(G)<\operatorname{Autcent}(G)$, which is again a contradiction. Hence the exponents of $\mathrm{Z}(G)$ and $\gamma_{2}(G)$ are equal. This completes the necessary part of the theorem.

Conversely suppose that $r=s,(G / \mathrm{Z}(G)) / \overline{\mathrm{M}} \cong\left(G / \gamma_{2}(G)\right) / \overline{\mathrm{N}}$ and the exponents of $\mathrm{Z}(G)$ and $\gamma_{2}(G)$ are equal. Since $r=s$, it follows that $G$ is purely non-abelian. For, suppose that $G$ is not purely non-abelian. Then $G=\mathrm{H} \times \mathrm{A}$, where A is nontrivial abelian and $\mathrm{H}$ is purely non-abelian subgroup of $G$. So $\gamma_{2}(G)=\gamma_{2}(\mathrm{H})$ and $\mathrm{Z}(G)=\mathrm{Z}(\mathrm{H}) \times \mathrm{A}$. This implies that

$$
\begin{aligned}
G / \gamma_{2}(G) & =G / \gamma_{2}(\mathrm{H})=(\mathrm{H} \times \mathrm{A}) / \gamma_{2}(\mathrm{H}) \cong\left(\mathrm{H} / \gamma_{2}(\mathrm{H})\right) \times\left(\mathrm{A} \gamma_{2}(\mathrm{H}) / \gamma_{2}(\mathrm{H})\right) \\
& \cong\left(\mathrm{H} / \gamma_{2}(\mathrm{H})\right) \times \mathrm{A}
\end{aligned}
$$

and

$$
\begin{aligned}
G / \mathrm{Z}(G) & =(\mathrm{H} \times \mathrm{A}) /(\mathrm{Z}(\mathrm{H}) \times \mathrm{A})=\mathrm{HZ}(\mathrm{H}) A / \mathrm{Z}(\mathrm{H}) A \cong \mathrm{H} /(\mathrm{H} \cap \mathrm{Z}(\mathrm{H}) A) \\
& =\mathrm{H} / \mathrm{Z}(\mathrm{H}) .
\end{aligned}
$$

These equations imply that the rank of $G / \gamma_{2}(G)$ is strictly greater than the rank of $\mathrm{H} / \gamma_{2}(\mathrm{H})$ and the rank of $G / \mathrm{Z}(G)$ is equal to the rank of $\mathrm{H} / \mathrm{Z}(\mathrm{H})$, where the rank of a finite abelian $p$-group $X$ is defined to be the number of non-trivial cyclic factors in a cyclic decomposition of $X$. Since the nilpotency class of $\mathrm{H}$ is 2 , we have $\gamma_{2}(\mathrm{H}) \subseteq \mathrm{Z}(\mathrm{H})$. So $\mathrm{H} / \mathrm{Z}(\mathrm{H})$ is a quotient group of $\mathrm{H} / \gamma_{2}(\mathrm{H})$. Thus it follows that the rank of $\mathrm{H} / \mathrm{Z}(\mathrm{H})$ is less than or equal to the rank of $\mathrm{H} / \gamma_{2}(\mathrm{H})$. Hence the rank of $G / \gamma_{2}(G)$ is strictly greater than the rank of $G / \mathrm{Z}(G)$. This implies that $s>r$, because $s$ is the rank of $G / \gamma_{2}(G)$ and $r$ is the rank of $G / \mathrm{Z}(G)$. This contradition proves that $G$ is purely non-abelian.

Since $r=s$ and $(G / \mathrm{Z}(G)) / \overline{\mathrm{M}} \cong\left(G / \gamma_{2}(G)\right) / \overline{\mathrm{N}}$, we have $G / \mathrm{Z}(G)=\mathrm{C}_{p^{a_{1}}} \times \mathrm{C}_{p^{a_{2}}}$ $\times \cdots \times \mathrm{C}_{p^{a_{s}}}$ and $G / \gamma_{2}(G)=\mathrm{C}_{p^{b_{1}}} \times \mathrm{C}_{p^{b_{2}}} \times \cdots \times \mathrm{C}_{p^{b_{s}}}$ such that $\mathrm{C}_{p^{a_{k+1}}} \times \cdots \times \mathrm{C}_{p^{a_{s}}}$ $\cong \mathrm{C}_{p^{b k+1}} \times \cdots \times \mathrm{C}_{p^{b_{s}}} \cong \mathrm{D}$ (say). So the smallest $t, 1 \leq t \leq s$ such that $a_{j}=b_{j}$ for all $j$ satisfying $t+1 \leq j \leq s$, is at the most $k$.

Now suppose that $\operatorname{Aut}_{\mathrm{Z}(G)}^{\mathrm{Z}(G)}(G)<\operatorname{Autcent}(G)$. Then by Lemma 2.1 (with $\mathrm{M}=$ $\mathrm{Z}(G))$ and Lemma 2.2. we have

$$
|\operatorname{Hom}(G / \mathrm{Z}(G), \mathrm{Z}(G))|<\left|\operatorname{Hom}\left(G / \gamma_{2}(G), \mathrm{Z}(G)\right)\right|,
$$


since $G$ is purely non-abelian. So it follows that $b_{j}>a_{j}$ for some $j$ such that $1 \leq j \leq s$ (to be more precise, it happens for some $j$ such that $1 \leq j \leq k$ ). For, if $b_{j}=a_{j}$ for all $j$, then $|\operatorname{Hom}(G / \mathrm{Z}(G), \mathrm{Z}(G))|=\left|\operatorname{Hom}\left(G / \gamma_{2}(G), \mathrm{Z}(G)\right)\right|$, which we are not taking. Now applying Lemma 2.5 with $\mathrm{A}=G / \mathrm{Z}(G), \mathrm{B}=G / \gamma_{2}(G)$ and $\mathrm{C}=\mathrm{Z}(G)$, we conclude that the exponent of $\mathrm{Z}(G)$ must be at least $p^{a_{t}+1}$, which is strictly bigger than $p^{a_{k}}=p^{c}$ - the exponent of $\gamma_{2}(G)$. This contradicts our supposition that $\mathrm{Z}(G)$ and $\gamma_{2}(G)$ have equal exponents. Hence $\operatorname{Aut}_{\mathrm{Z}(G)}^{\mathrm{Z}(G)}(G)=$ Autcent $(G)$, which completes the proof of the theorem.

It is fairly easy to deduce the following corollary from Proposition 2.3 and the theorem.

Corollary 2.6. Let $G$ be a non-abelian finite p-group. Then $\operatorname{Autcent}(G)=\operatorname{Inn}(G)$ if and only if $\mathrm{Z}(G)=\gamma_{2}(G)$ and $\mathrm{Z}(G)$ is cyclic.

Remark 2.7. It is of interest to find necessary and sufficient conditions for a finite $p$-group $G$ of arbitrary nilpotency class such that $\operatorname{Autcent}(G)=\operatorname{Aut}_{\mathrm{Z}(G)}^{\mathrm{Z}(G)}(G)$.

Acknowledgements. I thank Prof. E. C. Dade for some useful discussion and the referee for his/her useful comments and suggestions.

\section{REFERENCES}

[1] J. E. Adney and T. Yen, Automorphisms of a p-group, Illinois J. Math. 9 (1965), 137-143.

[2] M. S. Attar, On central automorphisms that fix the central elementwise, Arch. Math. 89 (2007), 296-297.

[3] J. N. S. Bidwell, M. J. Curran and D. J. McCaughan, Automorphisms of direct products of finite groups, Arch. Math. 86 (2006), 481-489.

[4] M. J. Curran and D. J. McCaughan, Central automorphisms that are almost inner, Comm. Algebra 29 (2001), 2081-2087.

[5] H. Liebeck, A note on prime power groups with symmetrical generating relations, Proc. Cambridge Philos. Soc. 51 (1955), 394-395.

School of Mathematics, Harish-Chandra Research Institute, Chhatnag Road, Jhunsi, Allahabad - 211 019, INDIA

E-mail address: myadav@hri.res.in 